\title{
Squamous cell carcinoma associated with Xeroderma pigmentosum: an unusual presentation with a tremendously huge mass over the face and paraneoplastic hypercalcemia- hyperleukocytosis
}

\author{
Suna Emir ${ }^{1}$, Şadan Hacısalihoğlu² ${ }^{2}$, Derya Özyörük ${ }^{3}$, Dilek Kaçar ${ }^{2}$, Arzu Erdem³, \\ Esra Karakuş ${ }^{4}$ \\ Department of ${ }^{1}$ Pediatric Oncology, Ankara LOSANTE Children's and Adult Hospital, Departments of ${ }^{2}$ Pediatric \\ Hematology Oncology, ${ }^{3}$ Pediatric Oncology, ${ }^{4}$ Pathology, Ankara Children's Hematology Oncology Training and Research \\ Hospital, Ankara, Turkey.E mail: sunaemir@yahoo.com \\ Received: 23th November 2016, Revised: 23th February 2017, Accepted: 27th February 2017
}

SUMMARY: Emir S, Hacısalihoğlu Ş, Özyörük D, Kaçar D, Erdem A, Karakuş E. Squamous cell carcinoma associated with Xeroderma pigmentosum: an unusual presentation with a tremendously huge mass over the face and paraneoplastic hypercalcemia-hyperleukocytosis. Turk J Pediatr 2017; 59: 711-714.

Xeroderma pigmentosum (XP) is a rare autosomal recessive disorder that results from genetic defects in DNA repair and manifests with a marked hypersensivity to ultraviolet rays. Children with X-P are at high risk of developing skin cancers.

On the other hand, hypercalcemia-hyperleukocytosis is a rare paraneoplastic syndrome in children with cancer compared to adults. Here, we report a five-year-old female with X-P and squamous cell carcinoma (SCC). The patient presented with a necrotic, ulcerating huge mass sized $20 \times 15 \times 10 \mathrm{~cm}$ involving the right half of the face. She had a history of increased freckling over the face since the age of two years. Her other cutaneous findings are dryness of skin, photosensitivity, freckling and telengiectasis all over the body. A diagnosis of Xeroderma pigmentosum was made based on clinical features. She also had high fever, anemia, hyperleukocytosis, thrombocytosis and hypercalcemia. After pathological diagnosis of squamous cell carcinoma, she was treated with chemotherapy. All the symptoms and signs resolved dramatically with the initiation of chemotherapy. Our case is an example of early development of massive disfiguring SCC in children with undiagnosed and untreated X-P. Although we could not prove the paraneoplastic nature of hypercalcemia-hyperleukocytosis, dramatic response to the chemotherapy may be an evidence for paraneoplastic origin.

Key words: squamous cell carcinoma, xeroderma pigmentosum, paraneoplastic syndrome, hypercalcemia, hyperleukocytosis.

Xeroderma pigmentosum (X-P) is a rare autosomal recessive disorder characterized with marked hypersensivity to ultraviolet rays. Due to defects in deoxyribonucleic acid (DNA) repair, photosensitivity, freckly pigmented changes and telengiectasis result. ${ }^{1-2}$ The risk of skin cancer is higher than normal population. There is a 10000 -fold increased risk of skin cancer on sun exposed sites. ${ }^{3-4}$ Squamous cell carcinoma (SCC) particularly involves the face, head, neck and scalp. It presents early and tends to be unusually aggressive in patients with X-P. Leukocytosis and hypercalcemia are two of the most common paraneoplastic syndromes associated with various malignancies in adults. However, it is a rare combination of paraneoplastic syndrome in children with cancer.

Herein, we report squamous cell carcinoma of the face in a five-year-old African female related 
to xeroderma pigmentosum who presented with a tremendously huge mass over the face and probable paraneoplastic-hypercalcemiahyperleukocytosis.

\section{Case Report}

A five-year-old African girl was referred to our country with the presumptive diagnosis of a facial tumor after she had been admitted to a local hospital in Somalia with the complaint of a rapidly growing mass over the right half of the face.

On admission, physical examination revealed a necrotic, ulcerating huge mass sized $20 \times 15 \times 10$ $\mathrm{cm}$ involving the right half of the face (Fig. $1)$. The right ear was pushed upwards due to the mass covering almost half of the face with foul smelling purulent discharge. This mass had been gradually progressing for the last two years. Her vital signs were stable except for high fever $\left(3^{\circ} \mathrm{C}\right)$. She had a history of increased freckling over the face since the age of two years. She had also multiple

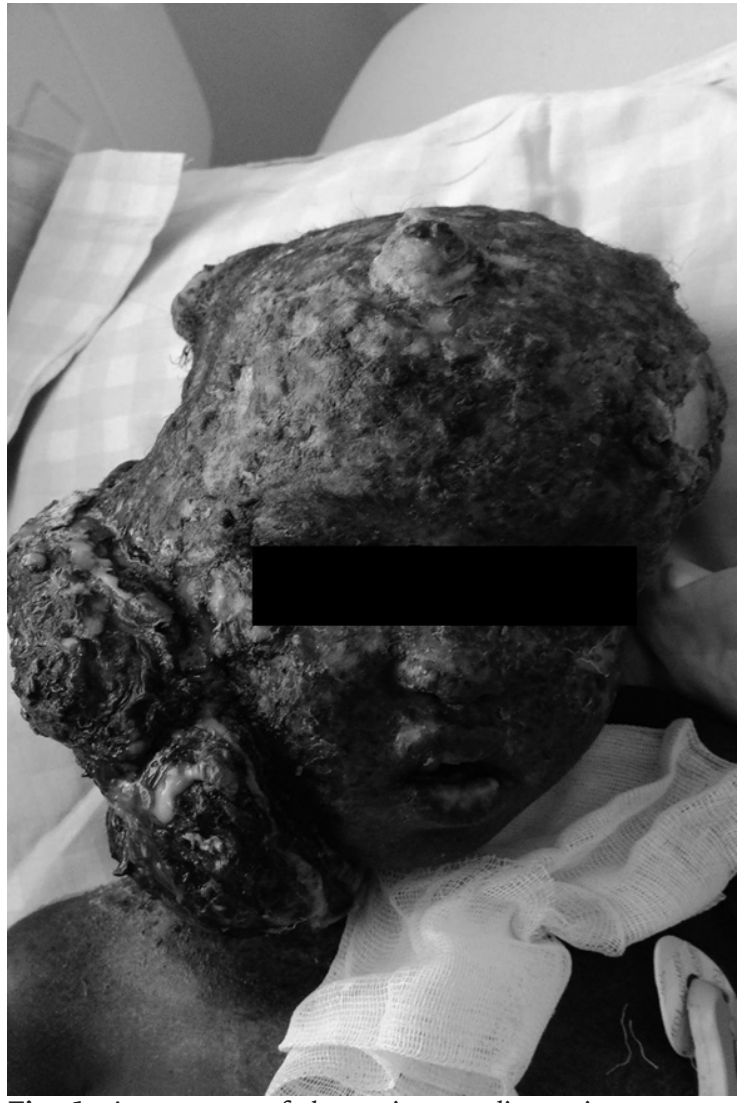

Fig. 1. Appearance of the patient at diagnosis nodular ulcerative lesions over the scalp. Her other cutaneous findings are dryness of skin, photosensivity, freckling and telengiectasis all over the body. Ocular examination showed ectropion, conjunctivitis, and keratitis. Her past history revealed that there was a first-degree consanguineous marriage between parents and sibling death from a similar disease. She was diagnosed with xeroderma pigmentosum according to her history and clinical findings. Complete blood count revealed the followings: $\mathrm{Hb} 5 \mathrm{~g} / \mathrm{dl}$, WBC $70000 / \mathrm{mm}^{3}$, platelet 797000/ $\mathrm{mm}^{3}$. She had a leukocyte count of 70000/ $\mathrm{mm}^{3}$ with predominance of neutrophils and band cells. Peripheral blood smears were consistent with a leukemoid reaction. All biochemical test results were within normal ranges except hypoalbuminemia, hypercalcemia and elevated C-reactive protein level (serum calcium $15 \mathrm{mg} / \mathrm{dl}$, phosphorus $2.7 \mathrm{mg} / \mathrm{dl}$. albumin $1.7 \mathrm{~g} / \mathrm{dl}$, C-reactive protein $8.9 \mathrm{mg} /$ dl) After blood, urine and pus cultures were obtained, she was started on broad spectrum intravenous antibiotics. Although antimicrobial therapy, intravenous hydration, and diuretics for hypercalcemia were started, response to the treatment was not achieved until 48 hours. The serum parathyroid hormone level was $3.5 \mathrm{pg} /$ $\mathrm{ml}$. Zoledronic acid at the dose of $0.1 \mathrm{mg} / \mathrm{kg}$ was given for hypercalcemia. Serum calcium levels decreased to normal levels within 72 hours. Cranial tomography showed that there was an erosion of the scalp exposing the

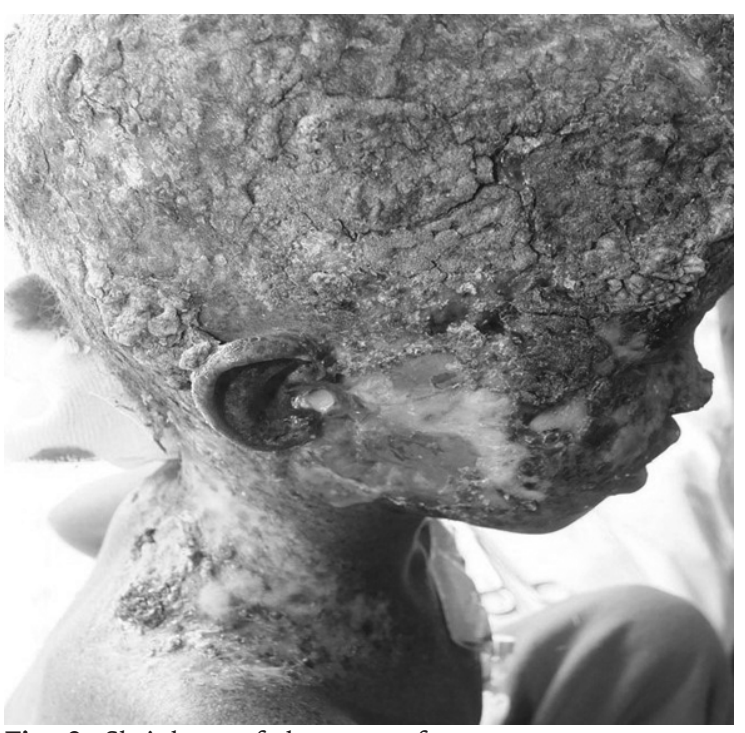

Fig. 2. Shrinkage of the mass after treatment 
dura with intracranial extension and a huge mass sized about $103 \times 50 \times 120 \mathrm{~mm}$ starting from the right temporaparietal region with irregular contours. She underwent incisional biopsy of the mass over the right ear which on histopathological examination was diagnosed as undifferentiated SCC. Chemotherapy consisting of Cisplatin and 5-fluorouracil was started. Paraneoplastic features resolved after one course of chemotherapy. The huge mass almost completely disappeared in subsequent courses. (Fig. 2.) Informed consent form has been obtained from parents for the publication of this case report.

\section{Discussion}

Xeroderma pigmentosum is a well known risk factor for skin cancers. ${ }^{1-2}$ The basic defect underlying the clinical findings is a nucleotide excision repair defect leading to a defective repair of DNA damaged by ultraviolet radiation. In children with XP, there is a 10000-fold increased risk of skin cancer under 20 years of age. The mean age for skin cancer is 8 years compared to 60 years in the healthy individuals. ${ }^{2-5}$ SCC of head and neck can present early and tends to be unusually aggressive in patients with X-P. Similarly, our patient was admitted with a very huge mass. At the time of admission, the presence of leukocytosis and fever were firstly considered as a result of infection. Despite the absence of improvement with the use of antibiotics, the possibility of a paraneoplastic syndrome came to mind.

Paraneoplastic syndromes are symptoms that occur at sites distant from a tumor and may be secondary to substances secreted by the tumor such as serum granulocyte colony stimulation factor (G-CSF) and interleukins. ${ }^{6-9}$ The data regarding hyperleukocytosis in patients with solid tumors is scarce. The incidence has been reported to range from $1 \%$ to $4 \% .^{5,6}$ If a patient with a solid tumor presents with leukocytosis, it is necessary to rule out secondary causes such as infections and use of corticosteroids. Paraneoplastic leukemoid reaction remains a diagnosis of exclusion. Granger et al. ${ }^{6}$ reported that they reviewed the etiology of hyperleukocytosis in 758 solid tumor patients. The etiology of the leukocytosis was the use of G-CSF in $69 \%$ of patients, infection in 112 , corticosteroids in 38 and newly diagnosed leukemia in 9 patients. Hypercalcemia-hyperleukocytosis paraneoplastic syndrome is rarely reported during the course of cutaneous squamous cell carcinoma.

Khawaja et al. ${ }^{10}$ reported that of the 35 patients who had hyperleukocytosis with bladder carcinoma, 9 of them also had hypercalcemia. Paraneoplastic hypercalcemia-leukocytosis is due to the paraneoplastic production of hematopoietic growth factors in a majority of these patients. Izard et al. ${ }^{7}$ reported that the incidence of paraneoplastic leukocytosis was $0,6 \%$ of their patients with urothelial carcinoma. They described nine patients with hyperleukocytosis with a median age of 63 years. Six of them also had hypercalcemia. They also reported that this paraneoplastic syndrome caused a poor prognosis in their patients. Said et al. ${ }^{11}$ reported two adult cases of paraneoplastic hyperleukocytosis and hypercalcemia complicating SCC. They supposed that this syndrome may be related to hormones or cytokines secretion by the neoplastic cells. Kato et al. ${ }^{12}$ described a 58-year-old man with SCC associated with hyperleukocytosishypercalcemia. His serum G-CSF and IL-1 levels were reported as high. They concluded that these humoral factors were responsible for the paraneoplastic syndrome induced by tumor cells in their case. Hiraki et al. ${ }^{9}$ investigated hypercalcemia, hyperleukocytosis and both in their 1149 patients with lung cancer. There were 65 cases of hypercalcemia, 16 cases of leukocytosis and six cases of both of them on admission. Survival of patients with the hypercalcemia-leukocytosis syndrome was significantly shorter than that of patients with hypercalcemia alone. They concluded that hypercalcemia-leukocytosis syndrome was very uncommon and an indicator for worse prognosis in patients with lung cancer. Presence of hypercalcemia and other paraneoplastic signs like leukemoid reaction is a potential marker for malignant behavior and poor prognosis in advanced tumors. We could not prove that the elevated white blood cells and calcium levels were paraneoplastic in nature. But, the dramatic response to the chemotherapy supported the probable diagnosis of paraneoplastic syndrome.

We speculate that our case highlights the presence of paraneoplastic hypercalcemia/ hyperleukocytosis associated with massive 
disfiguring SCC in a child with undiagnosed and untreated X-P.

\section{REFERENCES}

1. Gül U, Kılıç A, Gönül M, Çakmak SK, Soylu S. Xeroderma pigmentosum: a Turkish series. Int J Dermatol 2007; 46: 1125-1128.

2. Chaudhary M, Jajoo SN, Agarwal R. Xeroderma pigmentosum: A case report of two siblings. J Immunodefic Disor 2012; 1: 2.

3. Awan BA, Alzanbagi H, Samargandi OA, Ammar H. Scalp Squamous cell carcinoma in Xeroderma pigmentosum. N Am Med Sci 2014; 6: 105-106.

4. Rahman T, Sharma JD, Krishnnatreya M, Kataki AC. Basaloid sguamous carcinoma of skin associated with xeroderma pigmentosum in an 8-year old-old child: A rare entity. Indian J Dermatol 2014; 59: 632-635.

5. Shankar R, Qureshi SS, Sugoor P, Kembhavi S, Yadav PS, Mukta R. Colossal squamous cell carcinoma of the face in a child with Xeroderma pigmentosum. J Indian Assoc Pediatr Surg 2014; 19: 185-186.

6. Granger JM, Kontoyiannis DP. Etiology and outcome of extreme leukocytosis in 758 nonhematologic cancer patients. Cancer 2009; 115: 3919-3923.
7. Izard JP, Gore JL, Mostaghel EA, Wright JL, Yu EY Persistent, unexplained leukocytosis is a paraneoplastic syndrome associated with a poor prognosis in patients with urothelial carcinoma. Clin Genitourin Cancer 2015; 13: e253-e258.

8. Doraiswamy VA Biboa J, Obafemi A, Goldschmid M. Leukocytosis and hypercalcemia: a rare combination of paraneoplastic features in squamous cell penile cancer. South Med J 2010; 103: 474-476.

9. Hiraki A, Ueoka H, Takata I, et al. Hypercalcemialeukocytosis syndrome associated with lung cancer. Lung Cancer 2004; 43: 301-307.

10. Khawaja MR, Bradford CA, Azar JM. Paraneoplastic leukocytosis: an unusual manifestation of squamous cel carcinoma of the urinary bladder. Oncology (Willinston Park) 2013; 27: 1297-1301.

11. Said B, Maitre S, Perrot JL, Labeille B, Cambazard F. Hypercalcemia-hyperleukocytosis paraneoplastic syndrome complicating cutaneous squamous cell carcinoma. Report of two cases. Rev Med Interne 2010; 31: 309-311.

12. Kato N, Yasukawa K, Onozuka T, Kimura K. Paraneoplastic syndromes of leukocytosis, thrombocytosis and hypercalcemia are occasionally seen in patients suffering from progressive malignant disorders. J Dermatol 1999; 26: $352-358$. 